\title{
Radio emission as a tracer of the accretion rate
}

\author{
Elmar Koerding* \\ School of Physics and Astronomy \\ University of Southampton \\ Southampton SO17 1BJ \\ $U K$ \\ E-mail: elmar@phys.soton.ac.uk
}

I present a method to estimate the accretion rate and jet power from the core radio luminosity of jet emitting sources. Several applications are highlighted: The bolometric luminosity of hard state objects as a function of the accretion rate is observed. The findings suggest inefficient accretion both in stellar and supermassive black holes. Secondly, all soft state black holes seem to populate a plane in the space defined by the black hole mass, accretion rate and characteristic time scale. Using the presented technique, this plane can be extended to hard state objects and neutron stars. Finally, I use the jet power estimate to study the kinetic power output of active galactic nuclei.

Bursts, Pulses and Flickering:Wide-field monitoring of the dynamic radio sky June 12-15 2007 Kerastari, Tripolis, Greece

\footnotetext{
${ }^{*}$ Speaker.
} 


\section{Introduction}

Measuring one of the most basic parameters of an accreting system, the accretion rate, is hard or often even impossible. Typically, one uses the bolometric luminosity as a measure of the accretion rate, where the bolometric luminosity is estimated from the X-ray flux over a very large energy band (e.g., 0.1-100 keV). Using this measure assumes that the radiative efficiency of the object is known or at least constant. For black holes, the current paradigm suggests that they are accreting inefficiently at low accretion rates and that the radiative efficiency is a function of the accretion rate (e.g.,[6]). Also the jet power of an object is hard to access, but it is usually assumed that it depends linearly on the accretion rate - if in a jet-emitting state (e.g., [7]). Here, we use the radio emission from the jet as a tracer of the accretion rate.

Black hole X-ray binaries (XRB) can be found in several distinct states (e.g. [20, 1]). The two main states are the hard state characterised by a hard power-law in the X-ray spectrum and the soft state, where the X-ray spectrum is dominated by multi-color blackbody emission. If a source is in the hard state one usually observes radio emission which is ascribed to a compact jet. In the soft state no jet is visible and the radio emission is quenched by at least a factor 50. Additionally there is the intermediate state (IMS) which can be divided into the hard IMS showing an unstable radio jet and the soft IMS where no jet is observed. When the source moves from the hard IMS to the soft IMS one usually observes a radio flare. The accretion state of a compact object is not directly determined by the accretion rate. Only at low accretion rates a $\mathrm{BH}$ is in a single state: the hard state. Above an accretion rate of $\sim 2 \%$ of the Eddington rate the source can be found any one state (hard, soft, IMS). It is not yet known which physical parameter governs the state of a BH. For slightly different definitions (especially for the IMSs) see [15].

\section{Accretion rate measure}

To obtain an accretion measure based on radio emission we first have to construct a relation between radio luminosity and accretion rates. Thus, we need to build a sample of jet emitting sources which have a known accretion rate.

As mentioned in the introduction, black holes accreting at low Eddington rates are likely to be radiatively inefficient. BHs only have steady jets in their hard state. For any given hard state observation it is likely that the source is inefficiently radiating and the bolometric luminosity cannot be used to estimate the accretion rate. Once a source is in the efficiently radiating soft state the jet is quenched. However, the bolometric luminosity of a black hole transient does not change significantly during the transition from the hard to the soft state (e.g., [23]). As the transition is fast compared to the normal outburst evolution, it is unlikely that the accretion rate changes significantly during the transition. Thus, hard state objects near their transition are likely accreting radiative efficiently. This is further supported by the findings that there may be a thin standard accretion disc in bright hard state objects [19]. For the brightest hard state objects we can therefore calculate the accretion rate from the bolometric luminosity. As we need to observe a state transition to ensure that our observation had a high radiative efficiency, we only have 5 data-points from 5 different outbursts $(2 \times$ GX 339-4, Cyg X-1, 1859+226, V404 Cyg). Additionally we included GRS $1915+105$, which is constantly accreting near its Eddington limit. 

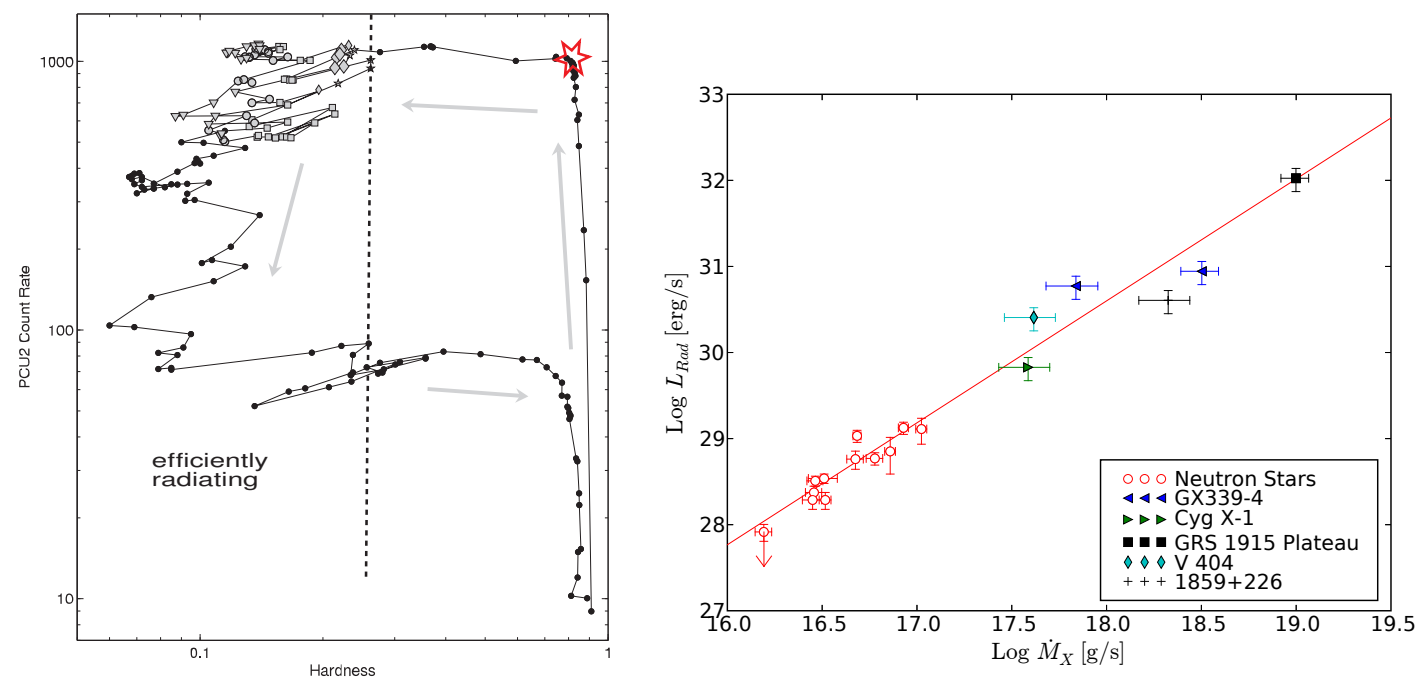

Figure 1: Left side: hardness intensity diagram for GX339-4 (reproduced with permission from Belloni et al 2005 [1]). The source is in the hard mostly inefficiently accreting state on the right side of the diagram. As we argue in the text we can only use the datapoint marked with the big red star to normalise our accretion rate measure. At this point the accretion flow needs to be efficiently radiating and still has a steady radio jet. Right side: Radio luminosity as a function of power liberated in the accretion disk for NSs and BHs.

Besides black hole objects atoll neutron stars (NSs) also seem to have steady radio emitting jets [18]. As they have a stellar surface, all accreted power has to be radiated away. They are therefore radiative efficient and the bolometric luminosity can be used to estimate the accretion rate. To compare NSs with BHs, we assume that the boundary layer contributes $1 / 2$ of the total bolometric luminosity [9]. This assumption is not crucial, see [12] for details.

The resulting correlation is shown in Fig. 1. All objects, neutron stars as well as black holes seem to follow the analytical prediction $L_{\text {rad }} \propto \dot{M}^{1.4}$ (e.g., [3]).

Thus, we can use the radio luminosity to estimate the accretion rate:

$$
\dot{M}=\dot{M}_{0}\left(\frac{L_{8 G H z}}{L_{0,8 G H z}}\right)^{0.71},
$$

where the normalisation factors $\left(\dot{M}_{0}, L_{0,8 G H z}\right)$ are determined by the normalisation of the radio / accretion rate correlation. As both constants are exchangeable we set $L_{0,8 G H z}=10^{30} \mathrm{erg} \mathrm{s}^{-1}$. This is roughly the $8.6 \mathrm{GHz}$ radio luminosity where the accretion disc around a $10 M_{\odot} \mathrm{BH}$ changes its spectral state. For $\dot{M}_{0}$ we find:

$$
\dot{M}_{0}=4.0 \times 10^{17} \mathrm{~g} \mathrm{~s}^{-1} .
$$

If one treats NSs and BHs separately and does not consider the contribution of the boundary layer, the normalisations for both classes differ by roughly a factor 2 (see [12]).

\section{Connection to variability properties}

As the accretion measure based on radio luminosities was constructed using only a few observations, it is important to check its validity using other measures of the accretion rate; here we 

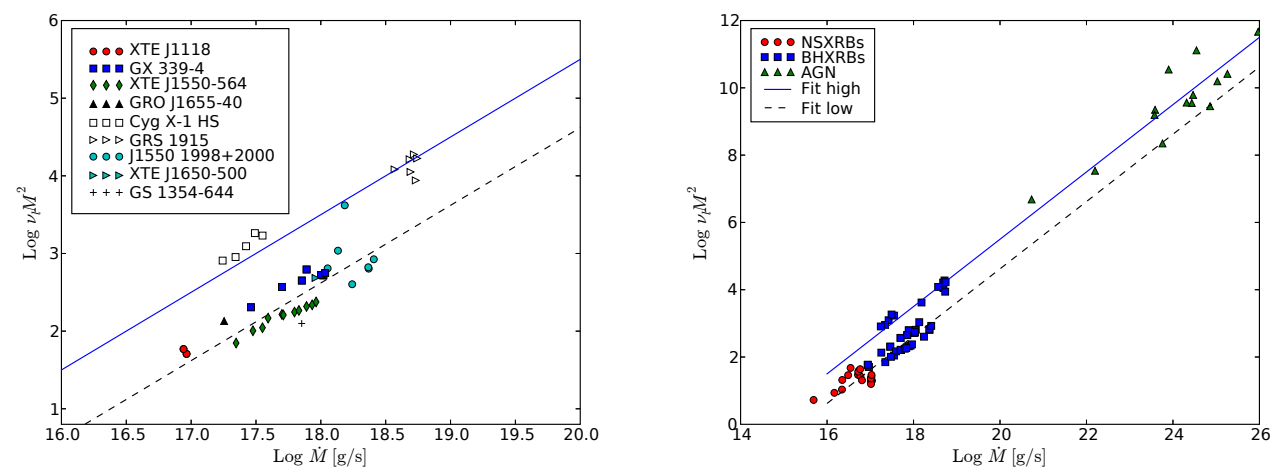

Figure 2: Left panel: Projection of the variability plane on the frequency, $\dot{M} M^{-2}$ plane. We show the correlation found for soft state black hole XRBs (solid line) as well as several hard state observations (fitted by the dashed line). The accretion rates of the hard state observations have been estimated using the described accretion measure. Right panel: Same projection as on the left panel. Here, we show the correlation for AGN, black hole XRBs and neutron stars with measured noise frequency $v_{l}$.

compare it to variability timescales that are thought to depend on the accretion rates as well [22]. The power spectral densities (PSD) of the X-ray light-curves of soft-state XRBs and AGN can be well described by a broken power law with a characteristic timescale corresponding to the break in the PSD. Recently, we found that these characteristic X-ray timescales not only depend on the black hole mass but also on the accretion rate. This allowed a direct connection between XRBs and AGN [16]: all soft state black holes lie on a plane in the space given by the characteristic frequency, the black hole mass and accretion rate.

The characteristic variability frequency depends linearly on the accretion rate in Eddington units and is inversely proportional on the black hole mass. Thus, we can compare our radio accretion rate measure with this independent accretion rate estimator.

The PSD of hard state X-ray binaries shows more features than those of a soft state object. It is usually fitted by a number of Lorentzians [2]. However, if one ignores the fine features of the PSD, the PSD for higher frequencies can also be described by a broken power law that declines a $v^{-2}$ at the high frequency end similar to the soft state case. We argue in [14] that one can identify the characteristic timescale found in AGN and soft state XRBs with the characteristic frequency $v_{l}$ of the lower high frequency Lorentzian.

We can now use our accretion measure based on the radio emission to calculate accretion rates for a sample of hard state black hole XRBs with measured frequencies $v_{l}$. In Fig. 2 we show the correlation between the characteristic frequencies and the black hole mass and accretion rate for soft state XRBs and AGN. Additionally, we show the sample of hard state XRBs. In both cases we find a linear correlation between the characteristic frequencies and the accretion rate.

There seems to be a constant offset between hard state objects and soft state objects. We also show two outbursts of XTE J1550 (1998 and 2000), where the source seem to be in a transition state. It starts off at the hard state scaling and moves towards the soft state scaling. Thus, the frequencies do not only depend on the accretion rates and black hole masses but also on a parameter governing the accretion state of the object. 

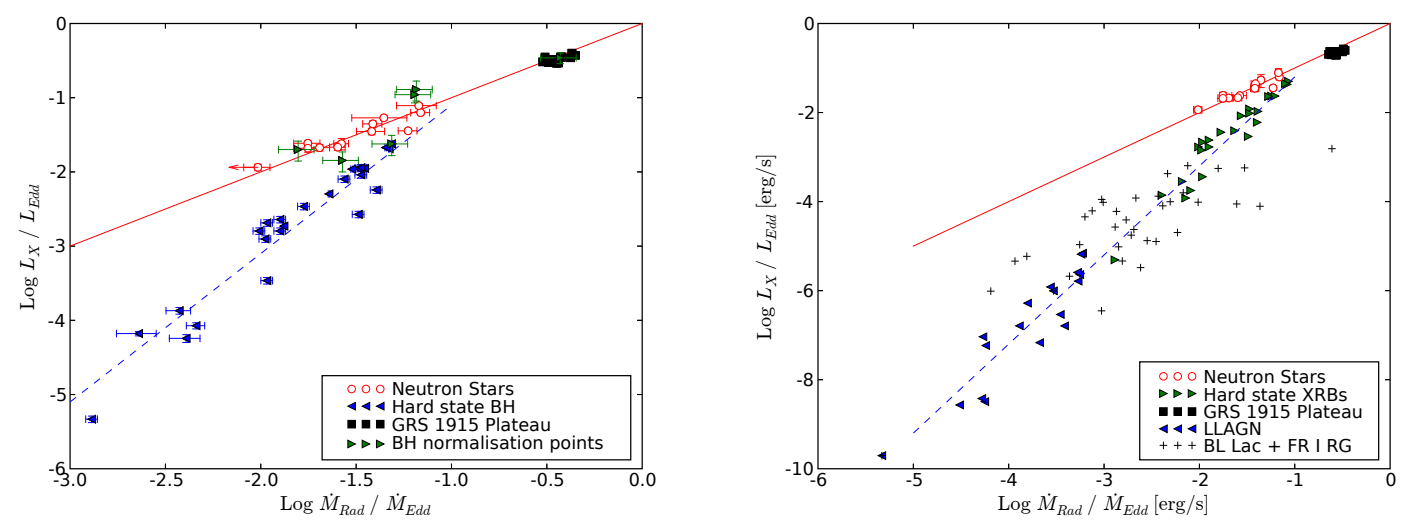

Figure 3: Bolometric luminosity as a function of the accretion rate estimated from the radio luminosity. Left side: Our sample of stellar BHs and NSs. While the bolometric luminosity in NSs depends linearly on accretion rate it scales quadratically for hard state BHs. Right side: Same as left only for AGN assuming the same bolometric correction for AGN as measured in BHs.

Besides black holes, one can also add neutron stars with measured frequencies $v_{l}$ of the noise components described by the lower high frequency Lorentzian. The resulting projection of the plane is shown on the right side of Fig. 2. We conclude that the accretion measure based on the radio emission is in agreement with the frequency/accretion rate relations.

\section{Bolometric luminosity}

As the accretion rate measure based on radio observations is not directly dependent on the bolometric luminosity, we can study the dependency of the bolometric luminosity on the accretion rate. We therefore construct a sample of radio emitting XRBs observations that have not been used to normalise the accretion measure.

In the left panel of Fig. 3 we show the bolometric luminosity as a function of the accretion rate estimated from radio luminosities. For NSs and efficiently accreting black holes (e.g., GRS 1915+105), we find a linear dependency of the bolometric luminosity on the accretion rate. However, many of those sources have been used to construct the accretion measure. This linear dependency simply reflects the fact that for NSs and efficiently accreting BHs the radio luminosity depends on the accretion rate as predicted by the conical jet models $\left(L_{r a d} \propto \dot{M}^{1.4}\right)$. However, the hard state black hole observations follow a different dependency: here the bolometric luminosity depends quadratically on the accretion rate. We note that this quadratic dependence does not depend on the normalisation of the accretion measure obtained from the radio observations of efficiently radiating BHs.

At very low accretion rates (of the order of $10^{-4} \dot{M}_{E d d}$ ) BHs have significantly lower bolometric luminosities than NSs ( $\sim$ two orders of magnitude). We can use this difference to explore if BHs advect some of the accreted power. All accreted power has to leave the accretion flow in some way. Energy can escape from the accreting system only in the form of matter (wind and jet) and as radiation. As we have shown, the power injected into the jet is similar for neutron stars and black holes for a given accretion rate. At such low accretion rate it is usually assumed that winds don't 
play a significant role. If we assume that winds do not carry a significant amount of energy from the system or if the winds are similar for NSs and black holes, we find that in black hole systems a significant fraction of the energy liberated in the accretion flow does not leave the system. It is therefore likely that this energy is advected into the black hole.

We can also use the radio accretion measure for AGN. If we directly use it, the X-ray luminosity of low luminosity AGN (LLAGN) lie significantly below the linear relationship found for efficiently radiating objects, already indicating that these (LLAGN) are likely inefficiently radiating like hard state objects. If one allows for a mild alteration of the accretion measure or introduces an 'effective bolometric correction' of the order of $M^{0.14}$, all AGN follow the quadratic correlation and we recover the 'fundamental plane of accreting black holes' [17, 8, 11]. In this formulation the fundamental plane is simply the quadratic relationship between bolometric luminosity and accretion rate found for inefficiently accreting objects.

\section{Accretion rate functions and jet powers}

The radio accretion measure seems also to be applicable also the AGN, if one can access the unbeamed radio luminosity. It is therefore possible to translate radio luminosity functions to accretion rate functions (ARFs). At low luminosity this is directly possible as low luminosity radio jets do not seem to be strongly beamed [4]. At high radio luminosities, i.e., for FR-II radio galaxies, the core radio flux is likely to be strongly beamed. However, low frequency radio emission coming from the lobes of the object can then be used to estimate the jet power and thus the accretion rate $[21,13]$.

In Fig. 4 we show the local bolometric luminosity function of AGN by [10]. The ARFs derived from LLAGN radio luminosity function and the ARF derived from $158 \mathrm{MHz}$ radio luminosities are shown as well. The difference of these different luminosity functions describes the fraction of 'radio-loud' objects. At low luminosities the LLAGN ARF is of a similar magnitude or even above the bolometric luminosity function of AGN. At high luminosities, there are significantly less radio loud objects than normal quasars. For details of the derivation of the ARF and the results see [13].

As we are estimating the accretion rate from jet properties, we can also derive the total kinetic power injected into the inter-galactic and inter stellar medium (IGM/ISM) by AGN jets. The total power injected by the different classes of AGN is shown in Fig. 4. In the local universe the total kinetic power injected into the ISM/IGM is dominated by low luminosity AGN and not by the rare radio loud quasars. Only at high redshifts is the so called 'quasar mode' feedback of similar value to the total power as LLAGN. In the quasar mode one assumes that $\sim 5 \%$ of the energy produced by the quasar is available for feedback ([5], the exact feedback mechanism is not yet fully established). Overall, the total kinetic power injected into the IGM by AGN is similar to that injected by supernovae.

EK acknowledges funding of a Marie Curie IEF under contract number MEIF-CT-2006024668. EK thanks Rob Fender, Tom Maccarone, Sebastian Jester, Simone Migliari, Ian $\mathrm{M}^{c} \mathrm{Hardy}$ and Phil Uttley for helpful discussions. 

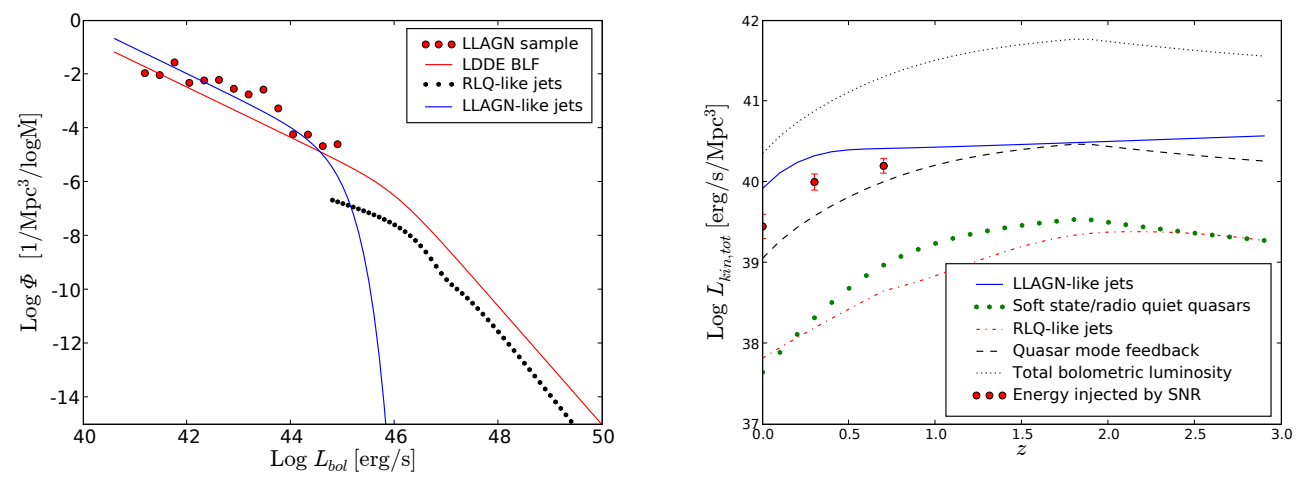

Figure 4: Left panel: Comparison of the accretion rate function derived from radio luminosities with a bolometric luminosity function (BLF) assuming luminosity dependend density evolution (LDDE). Right panel: Total kinetic power injected into the ISM/IGM by jets from AGN of different luminosity. In the local Universe the jets of low luminosity AGN (LLAGN) dominate the total energy input. Only at high redshifts are they comparable to the energy input due to quasars (assuming $5 \%$ efficiency). We also show the kinetic energy injected by radio loud quasars (RLQs).

\section{References}

[1] T. Belloni, J. Homan, P. Casella, M. van der Klis, E. Nespoli, W. H. G. Lewin, J. M. Miller, and M. Méndez, The evolution of the timing properties of the black-hole transient GX 339-4 during its 2002/2003 outburst, A\&A 440, 207-222 (2005).

[2] T. Belloni, D. Psaltis, and M. van der Klis, A Unified Description of the Timing Features of Accreting $X$-Ray Binaries, ApJ 572, 392-406 (2002).

[3] R. D. Blandford and A. Königl, Relativistic jets as compact radio sources, ApJ 232, 34-48 (1979).

[4] M. H. Cohen, M. L. Lister, D. C. Homan, M. Kadler, K. I. Kellermann, Y. Y. Kovalev, and R. C. Vermeulen, Relativistic Beaming and the Intrinsic Properties of Extragalactic Radio Jets, ApJ 658, 232-244 (2007) [arXiv: astro-ph/0611642].

[5] T. Di Matteo, V. Springel, and L. Hernquist, Energy input from quasars regulates the growth and activity of black holes and their host galaxies, Nature 433, 604-607 (2005) [astro-ph/0 02199 ].

[6] A. A. Esin, J. E. McClintock, and R. Narayan, Advection-dominated Accretion and the Spectral States of Black Hole X-Ray Binaries: Application to Nova MUSCAE 1991, ApJ 489, 865+ (1997).

[7] H. Falcke and P. L. Biermann, The jet-disk symbiosis. I. Radio to X-ray emission models for quasars., A\&A 293, 665-682 (1995).

[8] H. Falcke, E. Körding, and S. Markoff, A scheme to unify low-power accreting black holes. Jet-dominated accretion flows and the radio/X-ray correlation, A\&A 414, 895-903 (2004).

[9] J. Frank, A. King, and D. J. Raine, Accretion Power in Astrophysics: Third Edition, Accretion Power in Astrophysics, by Juhan Frank and Andrew King and Derek Raine, pp. 398. ISBN 0521620538. Cambridge, UK: Cambridge University Press, February 2002., February 2002.

[10] P. F. Hopkins, G. T. Richards, and L. Hernquist, An Observational Determination of the Bolometric Quasar Luminosity Function, ApJ 654, 731-753 (2007) [astro-ph / 0605678 ]. 
[11] E. Körding, H. Falcke, and S. Corbel, Refining the fundamental plane of accreting black holes, A\&A 456, 439-450 (2006) [astro-ph/0603117].

[12] E. G. Körding, R. P. Fender, and S. Migliari, Jet-dominated advective systems: radio and X-ray luminosity dependence on the accretion rate, MNRAS 369, 1451-1458 (2006).

[13] E. G. Körding, S. Jester, and R. Fender, Measuring the accretion rate and kinetic luminosity functions of supermassive black holes, MNRAS, 1100-(2007) [arXiv: 0710.1718].

[14] E. G. Körding, S. Migliari, R. Fender, T. Belloni, C. Knigge, and I. McHardy, The variability plane of accreting compact objects, MNRAS 380, 301-310 (2007) [arXiv: 0706 .2959].

[15] J. McClintock and R. Remillard, in "Compact Stellar X-ray Sources," eds. W.H.G. Lewin and M. v an der Klis, Cambridge University Press (2006).

[16] I. M. McHardy, E. Koerding, C. Knigge, P. Uttley, and R. P. Fender, Active galactic nuclei as scaled-up Galactic black holes, Nature 444, 730-732 (2006) [astro-ph/0612273].

[17] A. Merloni, S. Heinz, and T. Di Matteo, A Fundamental Plane of black hole activity, MNRAS 345, 1057-1076 (2003).

[18] S. Migliari and R. P. Fender, Jets in neutron star X-ray binaries: a comparison with black holes, MNRAS 366, 79-91 (2006) [astro-ph/0510698].

[19] J. M. Miller, J. Homan, D. Steeghs, M. Rupen, R. W. Hunstead, R. Wijnands, P. A. Charles, and A. C. Fabian, A Long, Hard Look at the Low/Hard State in Accreting Black Holes, ApJ 653, 525-535 (2006) [arXiv:astro-ph/0602633].

[20] M. A. Nowak, Toward a Unified View of Black-Hole High-Energy States, PASP 107, 1207+ (1995).

[21] S. Rawlings and R. Saunders, Evidence for a common central-engine mechanism in all extragalactic radio sources, Nature 349, 138-140 (1991).

[22] M. van der Klis, A Possible Explanation for the "Parallel Tracks" Phenomenon in Low-Mass X-Ray Binaries, ApJ 561, 943-949 (2001).

[23] S. N. Zhang, W. Cui, B. A. Harmon, W. S. Paciesas, R. E. Remillard, and J. van Paradijs, The 1996 Soft State Transition of Cygnus X-1, ApJ 477, L95 (1997). 